\title{
Site Specific Focused Ion Beam (FIB) Sample Preparation of Penetrative Oxidation in Ni-Base Alloys for 3DAPT Analysis
}

\author{
M.J. Olszta, L.E. Thomas and S.M. Bruemmer
}

Energy and Environment Directorate, Pacific Northwest National Laboratory, PO Box 999, Richland, WA 99352

A unique penetrative oxidation phenomenon has been observed in stress corrosion cracks for high $\mathrm{Cr}$, Ni-base alloys tested in high-temperature hydrogenated water. Conventional corrosion knowledge of these high $\mathrm{Cr}$ alloy would suggest that a continuous, protective, Cr-rich oxide layer would form along the crack walls. The penetrative oxidation can be seen on highly polished crosssections (Figure 1) using low $\mathrm{kV}$, high-resolution scanning electron microscopy (SEM) with a low angle backscatter electron detector (LABE). Transmission electron microscopy (TEM) observations have shown that these penetrations consist of two nanometer-scale, oriented crystalline phases, MOstructure oxide particles and $\mathrm{Cr}_{2} \mathrm{O}_{3}$ platelets. Due to the size scale, density and close physical relationship of these two oxides, understanding their composition and spatial relationships is difficult using TEM.

In order to fully elucidate the microstructure and chemistry of this penetrative oxidation, site specific sample preparation for three-dimensional atom probe tomography (APT) using the focused ion beam (FIB) is necessary. The three-dimensional relationship and composition provided by APT could then be used in correlation with crystallographic data collected via TEM to fully describe this penetrative oxidation process. This paper outlines procedures for preparing FIB lift-out APT tips of the penetrative oxidation.

Highly polished cross-section samples of the cracked region were first examined using low kV SEM to identify the extent of penetrative oxidation. APT tips were extracted by FIB normal to the crack growth direction as indicated in Figure 1 to determine the oxidation characteristics as a function of depth into the alloy matrix. A protective layer of Pt was deposited along the cross-section of the crack wall, and trenches were subsequently milled around the crack wall (Figure 2a). Once the sample was fully undercut, it was lifted out from the cross-section and then rotated $90^{\circ}$ such that any crack wall oxide would be positioned at the top of the APT tip (Figure 2b). The final APT tip was fashioned such that the very edge of the crack wall was at the top of the tip with the penetrative oxidation structures below (Figure 2c).

Figure 3 illustrates a typical data set from atomic reconstructions of the filamentary penetrative oxidation and the remaining metallic alloy. Near the crack wall surface, the metal has been nearly completely oxidized. Deeper in to the sample, the oxide penetrations can be observed as discrete filaments in the metal matrix. This sample preparation protocol is being used to assess penetrative oxidation morphologies in these Ni-Cr alloys both from crack walls and from surfaces. Site-specific FIB preparation is a critical step enabling high-resolution TEM and APT investigations of oxidation mechanisms that control corrosion and stress corrosion behavior. 


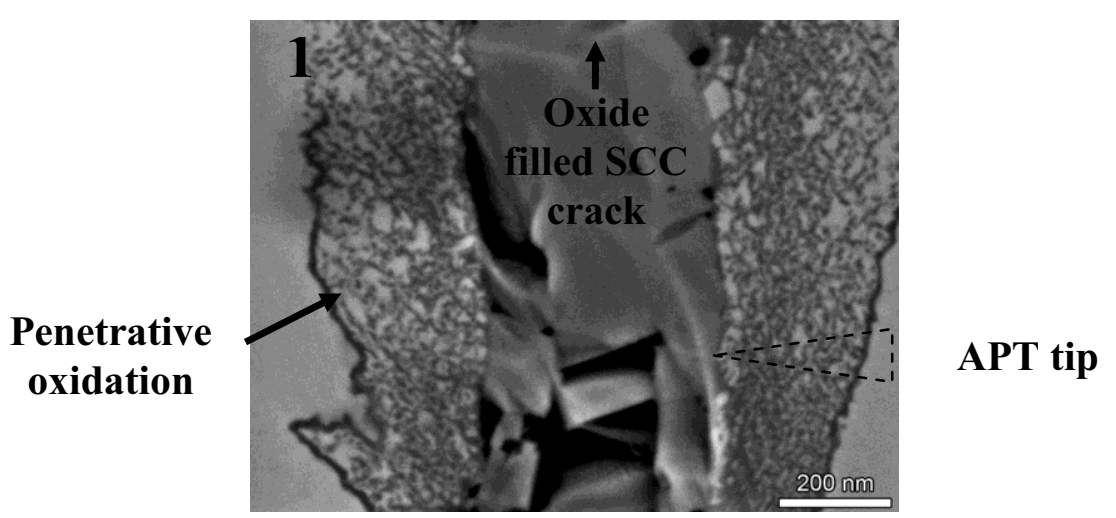

FIG. 1. SEM-LABE micrograph of a SCC crack with penetrative oxidation along the either side of the crack wall. The location and orientation of an APT tip is represented by a dashed triangle.

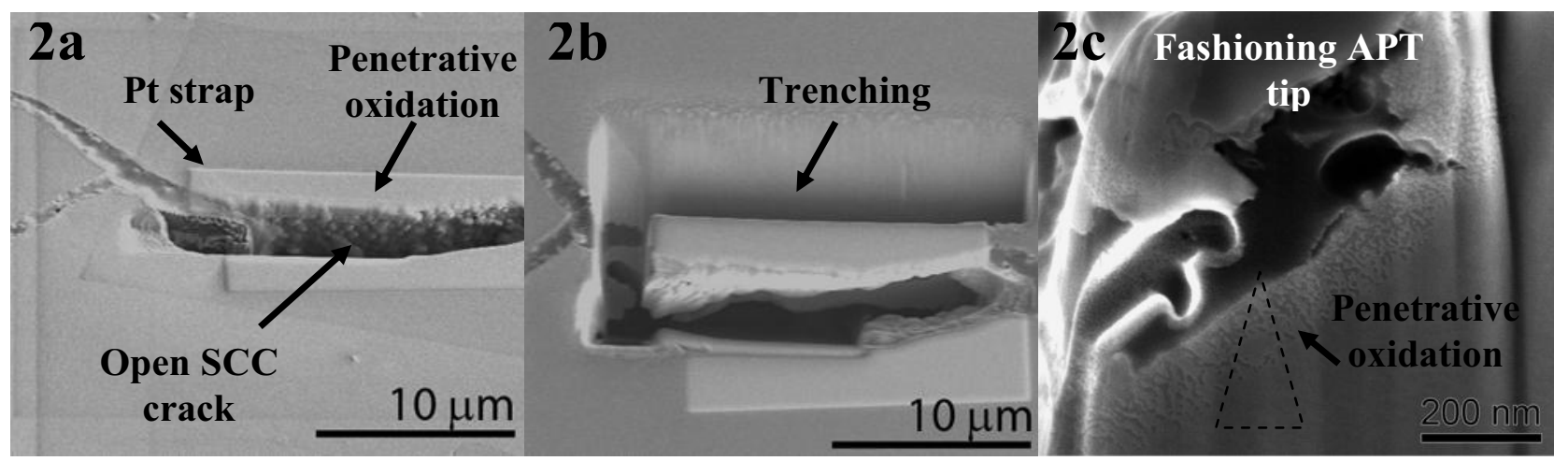

FIG. 2. Scanning electron micrographs (SE) illustrating the FIB lift-out procedure for procuring APT tips from penetrative oxidation along SCC crack walls. a) Region of interest is protected with Pt. b) Trenches are milled around the sample, and sample is lifted out and rotated (not shown). c) Fashioning of the APT tip with penetrative oxidation oriented with the crack wall at the top of the tip (dashed triangle illustrates final tip shape).

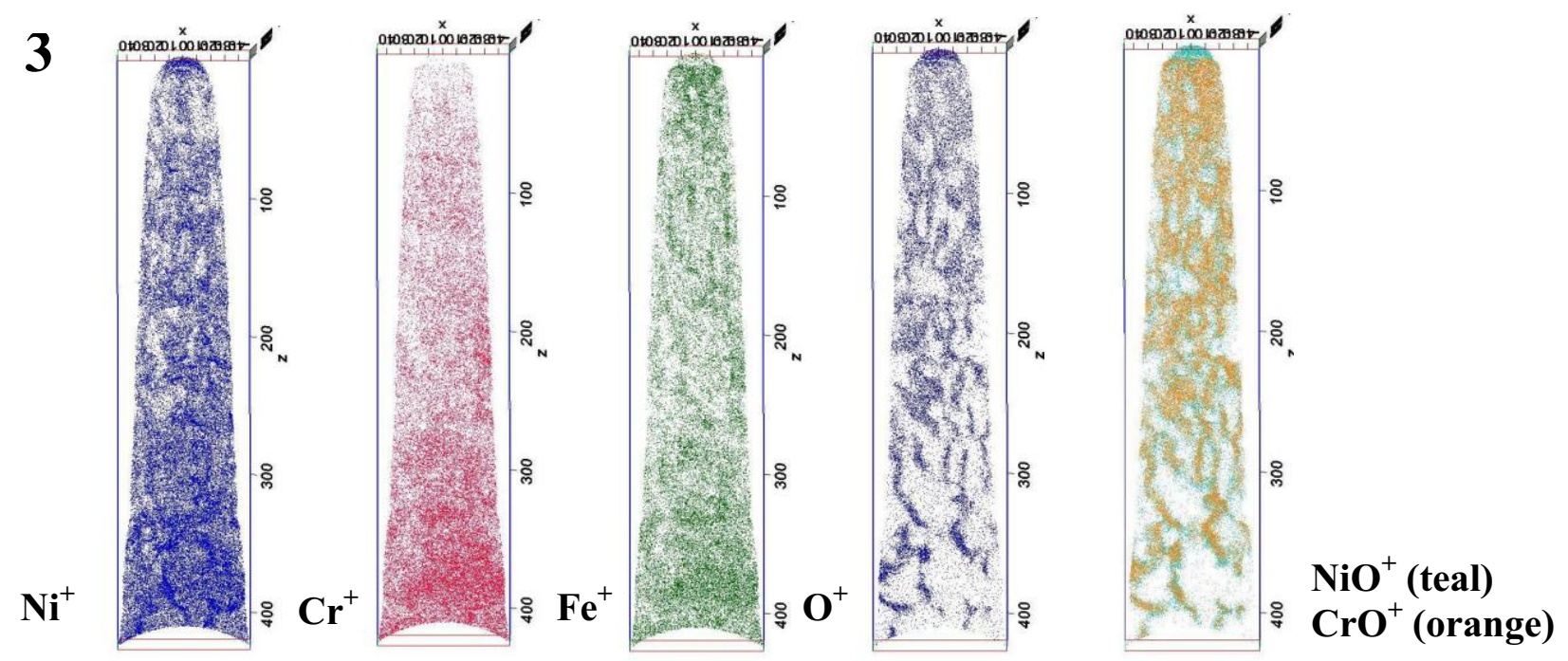

FIG. 3. 3DAPT reconstructions of $\mathrm{Ni}, \mathrm{Cr}, \mathrm{Fe}, \mathrm{O}, \mathrm{NiO}^{+}$and $\mathrm{CrO}^{+}$. Each atom map represents a $10 \mathrm{~nm}$ slice through the center of the APT sample (all measurements in $\mathrm{nm}$ ). 